\title{
Thermodynamic assessment of the Pd-Y binary system
}

\author{
S. Kardellass, N. Selhaoui, A. Iddaoudi, M. Ait Amar, R. Karioui, and L. Bouirden \\ Laboratory of Thermodynamics and Energy (L.T.E), Faculty of science, BP. 8106, University IbnZohr, Agadir, \\ Morocco
}

\begin{abstract}
The Pd-Y system was critically assessed using the CALPHAD technique. The solution phases (liquid, b.c.c., f.c.c. and h.c.p.) were modeled using the Redlich-Kister equation. The intermetallic compounds $\mathrm{Pd}_{3} \mathrm{Y}$ and $\mathrm{PdY}$, which have homogeneity ranges, were treated as the formula $(\mathrm{Pd}, \mathrm{Y})_{0.75}(\mathrm{Pd}, \mathrm{Y})_{0.25}$ and $(\mathrm{Pd}, \mathrm{Y})_{0.5}(\mathrm{Pd}, \mathrm{Y})_{0.5}$ by a two-sublattice model with a mutual substitution of $\mathrm{Pd}$ and $\mathrm{Y}$ on both sublattices. The optimization was carried out in two steps. In the first treatment, $\mathrm{Pd}_{3} \mathrm{Y}$ and PdY are assumed to be stoichiometric compounds; in the second treatment they are treated by a sublattice model. The parameters obtained from the first treatment were used as starting values for the second treatment. The calculated phase diagram and the thermodynamic properties of the system are in satisfactory agreement with the experimental data.
\end{abstract}

\section{INTRODUCTION}

The rare earth elements exhibiting a good combination of magnetic, optical, electrical and thermal properties have been paid increasing attention and widely used to prepare new high performance functional materials, such as permanent magnet, magnetic refrigerant and hydrogen storage material [1-3]. The CALPHAD technique, which has been recognized to be an important tool to significantly reduce time and cost during the development of materials, can provide a clear guidance for the materials design [4-6]. In order to develop the thermodynamic database of the rare earth alloys for reliable predictions of liquidus, phase fraction, equilibrium and non-equilibrium solidification behavior, etc. The thermodynamic assessment of the Pd-Y binary system was carried out by means of the CALPHAD method.

\section{REVIEW OF EXPERIMENTAL DATA}

The phase diagram of the Pd-Y system was first measured by Loebich and Raub [7]. They found seven intermetallic compounds in the $\mathrm{Pd}-\mathrm{Y}$ system and called them $\mathrm{Pd}_{3} \mathrm{Y}$, $\mathrm{Pd}_{2} \mathrm{Y}, \mathrm{Pd}_{5} \mathrm{Y}_{4}, \mathrm{PdY}, \mathrm{PdY}_{3}, \mathrm{Pd}_{3} \mathrm{Y}_{2}$ and $\mathrm{Pd}_{2} \mathrm{Y}_{5}$, where $\mathrm{Pd}_{3} \mathrm{Y}$, $\mathrm{PdY}$ and $\mathrm{Pd}_{5} \mathrm{Y}_{4}$ melted congruently and $\mathrm{Pd}_{3} \mathrm{Y}_{2}, \mathrm{Pd}_{2} \mathrm{Y}$ and $\mathrm{Pd}_{2} \mathrm{Y}_{3}$ formed by peritectic reactions. Later, $\mathrm{Pd}_{5} \mathrm{Y}_{4}$ was identified to be $\mathrm{Pd}_{4} \mathrm{Y}_{3}$ by Palenzona and Iandelli [8]. $\mathrm{Pd}_{7} \mathrm{Y}$ and $\mathrm{PdY}_{3}$ were found to exist by Sanjines-Zeballos et al. [9] and Takao et al. [10], respectively. The results obtained have been taken by Massalski [22] to draw a phase diagram. Kuentzler and Loebich [11] presented a revised phase diagram and discussed the stability of the existing ordered structures. The homogeneity range of the intermetallic compound $\mathrm{Pd}_{3} \mathrm{Y}$ and the solubility of $\mathrm{Y}$ in f.c.c.(Pd) were measured by Loebich and Raub [7] and Harris and Norman [12]. No appreciable solid solubility of $\mathrm{Pd}$ in $\mathrm{Y}$ was observed [7]. Loebich and Raub [7] indicated the presence of polymorphic transformation and a small range of homogeneity for PdY (51-52 at.\% Y). Considering, however, the subsequent work of Kuentzler and Loebich [11] and Palenzona and Cirafici [13], the homogeneity range of PdY should be 50-51 at.\% Y and the maximum melting point attributed to the ideal 50 at.\% Y composition [14].

\section{THERMODYNAMIC MODELS}

\subsection{Pure elements}

The Gibbs energy function

$$
\mathrm{G}_{\mathrm{i}}(\mathrm{T})={ }^{\circ} \mathrm{G}_{\mathrm{i}}-\mathrm{H}_{i}^{\mathrm{SER}}(298.15 \mathrm{~K})
$$

(298.15) for the element $\mathrm{i}(\mathrm{i}=\mathrm{Pd}, \mathrm{Y})$ in the phase $\varphi(\varphi=$ Liquid, HCP_A3, BCC_A2 and FCC_A1) is described by an equation of the following form:

$$
\begin{aligned}
\mathrm{G}_{\mathrm{i}}(\mathrm{T})= & \mathrm{a}+\mathrm{bT}+\mathrm{cT} \ln \mathrm{T}+\mathrm{dT}^{2}+\mathrm{eT}^{3}+\mathrm{fT}^{7} \\
& +\mathrm{gT}^{-1}+\mathrm{hT}^{-9} .
\end{aligned}
$$

Where $\mathrm{H}_{\mathrm{i}}^{\mathrm{SER}}(298.15 \mathrm{~K})$ is the molar enthalpy of the element $\mathrm{i}(\mathrm{i}=\mathrm{Pd}, \mathrm{Y})$ at $298.15 \mathrm{~K}$ in its standard element reference (SER) state, FCC_A1 for Pd and HCP_A3 for Y.

In this article, the Gibbs energy functions are taken from the SGTE compilation of Dinsdale [15].

\subsection{Solution phases}

The solution phases [(FCC_A1) Pd, HCP_A3 $(\alpha \mathrm{Y})$, BCC_A2 $(\beta \mathrm{Y})$ and liquid] were modeled as substitutional solutions according to the polynomial Redlich-Kister model [16]. The Gibbs energy of one mol of formula unit of phase $\varphi$ is expressed as the sum of the reference part ${ }^{\text {ref }} G^{\varphi}$, the ideal part ${ }^{\text {id }} G^{\varphi}$, and the excess part ${ }^{\text {exc }} G^{\varphi}$ :

$$
\mathrm{G}_{\mathrm{m}}^{\varphi}={ }^{\mathrm{ref}} G^{\varphi}+{ }^{\mathrm{id}} G^{\varphi}+{ }^{\mathrm{exc}} G^{\varphi} .
$$

As used in the thermo-calc software [17]:

$$
\begin{aligned}
{ }^{\text {ref }} G^{\varphi}(\mathrm{T})= & \left({ }^{0} \mathrm{G}_{\mathrm{Pd}}^{\varphi}(\mathrm{T})-\mathrm{H}_{\mathrm{Pd}}^{\mathrm{SER}}(298.15 \mathrm{~K})\right) \mathrm{x}_{\mathrm{Pd}} \\
& +\left({ }^{0} \mathrm{G}_{\mathrm{Y}}^{\varphi}(\mathrm{T})-\mathrm{H}_{\mathrm{Y}}^{\mathrm{SER}}(298.15 \mathrm{~K})\right) \mathrm{x}_{\mathrm{Y}}
\end{aligned}
$$




$$
{ }^{\mathrm{id}} G^{\varphi}=\mathrm{RT}\left(\mathrm{x}_{\mathrm{Pd}} \ln \mathrm{x}_{\mathrm{Pd}}+\mathrm{x}_{\mathrm{Y}} \ln \mathrm{X}_{\mathrm{Y}}\right)
$$

Where $\mathrm{R}$ is the gas constant, $\mathrm{T}$ the temperature, in Kelvin, Warning: $\mathrm{X}_{\mathrm{Pd}}$ and $\mathrm{x}_{\mathrm{Y}}$ are the fraction of elements $\mathrm{Pd}$ and $\mathrm{Y}$, respectively.

The excess terms of all the solution phases were modeled by the Redlich-Kister [16] formula:

$$
\begin{gathered}
{ }^{e x c} G_{m}^{\varphi}(T)=x_{\mathrm{Pd}} x_{\mathrm{Y}}\left[{ }^{0} L_{\mathrm{Pd}, \mathrm{Y}}^{\varphi}(T)+{ }^{1} \mathrm{~L}_{\mathrm{Pd}, \mathrm{Y}}^{\varphi}(T)\left(x_{\mathrm{Pd}}-x_{\mathrm{Y}}\right)\right. \\
\left.+L_{\mathrm{Pd}, \mathrm{Y}}^{\varphi}(T)\left(x_{\mathrm{Pd}}-x_{\mathrm{Y}}\right)^{2}+\cdots\right] \\
{ }^{i} \mathrm{~L}_{\mathrm{Pd}, \mathrm{Y}}^{\varphi}(T)=a_{i}+b_{i} T .
\end{gathered}
$$

Where ${ }^{i} \mathrm{~L}_{\mathrm{Pd}, \mathrm{Y}}^{\varphi}(\mathrm{T})$ is the interaction parameter between the elements Pd and Y, which is evaluated in the presented work, $\mathrm{a}_{i}$ and $\mathrm{b}_{i}$ are the coefficients to be optimized.

\subsection{Stoichiometric compounds and intermediate phases}

The Gibbs energy of the stoichiometric compounds $A_{p} B_{q}$ is expressed as follows:

$$
{ }^{0} G_{A_{p} B_{q}}=\frac{p}{p+q}{ }^{0} G_{A}+\frac{q}{p+q}{ }^{0} G_{B}+a+b T .
$$

Where ${ }^{0} G_{A}$ and ${ }^{0} G_{B}$ are the Gibbs energy of the pure elements $\mathrm{Pd}$ and $\mathrm{Y}$, respectively, $\mathrm{a}$ and $\mathrm{b}$ are parameters to be determined.

The $\mathrm{Pd}_{3} \mathrm{Y}\left(\mathrm{C} 14\right.$ Laves, isotypic with $\left.\mathrm{AuCu}_{3}\right)$ and PDY are treated by a two-sublattice model $[18,19]$. The Gibbs energy function per mole $(\mathrm{m})$ of the formula unit is the following:

$$
\begin{aligned}
G_{m}^{\mathrm{Pd} 3 \mathrm{Y}}= & y_{\mathrm{Pd}}^{\prime} y_{\mathrm{Pd}}^{\prime \prime}{ }^{0} G_{\mathrm{Pd}: \mathrm{Pd}}+y_{\mathrm{Pd}}^{\prime} y_{\mathrm{Y}}^{\prime \prime} G_{\mathrm{Pd}: \mathrm{Y}}+y_{\mathrm{Y}}^{\prime} y_{\mathrm{Pd}}^{\prime \prime} G_{\mathrm{Y}: \mathrm{Pd}} \\
& +y_{\mathrm{Y}}^{\prime} y_{\mathrm{Y}}^{\prime \prime} G_{\mathrm{Y}: \mathrm{Y}}+p R T\left(y_{\mathrm{Pd}}^{\prime} \operatorname{Ln} y_{\mathrm{Pd}}^{\prime}+y_{\mathrm{Y}}^{\prime} \operatorname{Ln} y_{\mathrm{Y}}^{\prime}\right) \\
& +p R T\left(y_{\mathrm{Pd}}^{\prime \prime} \operatorname{Ln} y_{\mathrm{Pd}}^{\prime \prime}+y_{\mathrm{Y}}^{\prime \prime} L n y_{\mathrm{Y}}^{\prime \prime}\right) \\
& +p y_{\mathrm{Pd}}^{\prime} y_{\mathrm{Y}}^{\prime}\left[y_{\mathrm{Pd}}^{\prime \prime} \sum_{n}{ }^{n} L_{\mathrm{Pd}, \mathrm{Y}: \mathrm{Pd}}\left(y_{\mathrm{Pd}}^{\prime}-y_{\mathrm{Y}}^{\prime}\right)^{n}\right. \\
& \left.+y_{\mathrm{Y}}^{\prime \prime} \sum_{n}{ }^{n} L_{\mathrm{Pd}, \mathrm{Y}: \mathrm{Y}}\left(y_{\mathrm{Pd}}^{\prime}-y_{\mathrm{Y}}^{\prime}\right)^{n}\right] \\
& +q y_{\mathrm{Pd}}^{\prime \prime} y_{\mathrm{Y}}^{\prime \prime}\left[y_{\mathrm{Pd}}^{\prime} \sum_{n}{ }^{n} L_{\mathrm{Pd}: \mathrm{Pd}, \mathrm{Y}}\left(y_{\mathrm{Pd}}^{\prime \prime}-y_{\mathrm{Y}}^{\prime \prime}\right)^{n}\right. \\
& \left.+y_{\mathrm{Y}}^{\prime} \sum_{n}{ }^{n} L_{\mathrm{Y}: \mathrm{Pd}: \mathrm{Y}}\left(y_{\mathrm{Pd}}^{\prime \prime}-y_{\mathrm{Y}}^{\prime \prime}\right)^{n}\right] \\
G_{m}^{\mathrm{PdY}}= & y_{\mathrm{Pd}}{ }^{0} G_{\mathrm{Pd}: \mathrm{Y}}+y_{\mathrm{Y}: \mathrm{Y}}{ }^{0} G_{\mathrm{Y}: \mathrm{Y}}+R T\left(y_{\mathrm{Pd}} L n y_{\mathrm{Pd}}\right) \\
& \left.+y_{\mathrm{Y}} L n y_{\mathrm{Y}}\right) \\
& +y_{\mathrm{Y}} \sum_{n}{ }^{n} L_{\mathrm{Pd}, \mathrm{Y}: \mathrm{Y}}\left(y_{\mathrm{Pd}}-y_{\mathrm{Y}}\right)^{n}
\end{aligned}
$$

Where $y_{i}^{\prime}$ and $y_{j}^{\prime \prime}$ are the site fraction of component $i$ and $j(i, j=\mathrm{Pd}, \mathrm{Y})$ located on sublattice I and II, respectively, and the parameter ${ }^{0} \mathrm{G}_{i: j}$ represents the Gibbs free energy of the compound phase when the two sublattices are
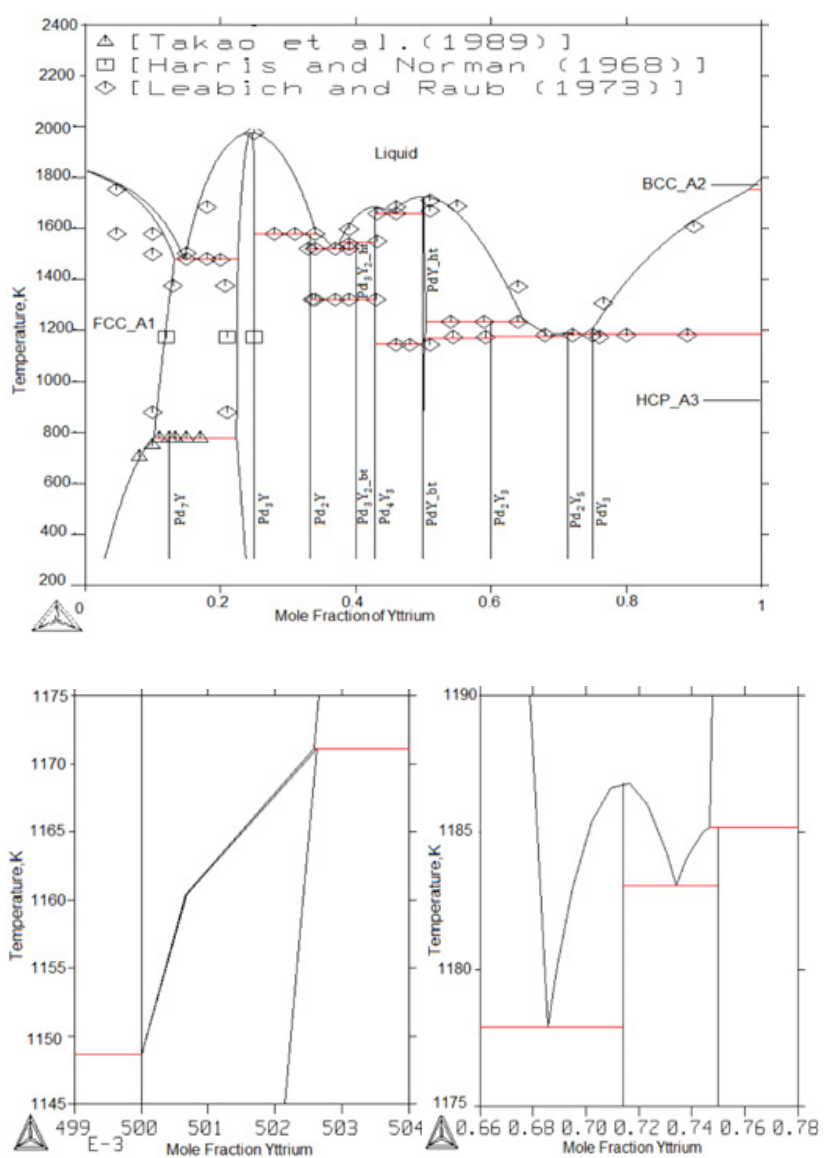

Figure 1. Comparison of the Pd-Y calculated phase diagram with the experimental data. (b) Enlarged section of (Fig. 1).

occupied by element $i$ or $j \cdot L_{i \cdot \mathrm{Pd} \cdot \mathrm{Y}}$ and $L_{\mathrm{PdY} \cdot j}$ are the interaction parameters between $\mathrm{Pd}$ and $\mathrm{Y}$ in the second or first sublattice, when the other sublattice is occupied by element $i$ or $j .{ }^{0} \mathrm{G}_{i, j}, \mathrm{~L}_{i: \mathrm{Pd}, \mathrm{Y}}$ and $\mathrm{L}_{\mathrm{Pd}, \mathrm{Y}: j}$ were evaluated in the present work.

\section{RESULTS AND DISCUSSIONS}

The evaluation of the phase diagram was carried out with the computer Thermo-Calcsoftoware [17] which makes it possible to optimize the formation Gibbs energies of the system phases by means of the least squares method using simultaneously thermodynamic and phase diagram data. The calculated phase diagram is shown in Fig. 1. It is compared with the numerous experimental data in Fig. 1. A good agreement is noted.

All the parameters were evaluated and listed in Table 1. For the intermetallic compounds $\mathrm{Pd}_{3} \mathrm{Y}, \mathrm{Pd}_{4} \mathrm{Y}_{3}, \mathrm{PdY}$ _ht, the coefficients $a$ and $b$ in Eq. (7), were adjusted according to the enthalpiesof formation measured by [22].

The calculated invariant equilibria in the Pd-Y system are listed in Table 2, where the largest uncertainty is about $4{ }^{\circ} \mathrm{C}$ in the invariant temperature of the reaction Liq $\leftrightarrow \mathrm{Pd}_{2} \mathrm{Y}_{5}+\mathrm{Pd}_{2} \mathrm{Y}_{3}$ In the reaction Liq $\leftrightarrow \mathrm{Pd}_{2} \mathrm{Y}_{5}+$ $\mathrm{Pd}_{2} \mathrm{Y}_{3}$, the uncertainty in the invariant temperature is about $13{ }^{\circ} \mathrm{C}$ [11]. In view of the estimated experimental 


\section{REMCES XII}

Table 1. The optimized thermodynamic parameters of the Pd-Y system.

\begin{tabular}{|c|c|c|}
\hline Phase & Thermodynamic models & Parameters in SI \\
\hline Liquid & $(\mathrm{Pd}, \mathrm{Y})_{1}$ & $\begin{array}{l}{ }^{0} \mathrm{~L}^{\text {liq }}=-344376.073+21.686 \mathrm{~T} \\
{ }^{1} \mathrm{~L}^{\text {liq }}=-209832.431+54.045 \mathrm{~T}\end{array}$ \\
\hline BCC_A2 & $(\mathrm{Pd}, \mathrm{Y})_{1}(\mathrm{Va})_{3}$ & No excess term \\
\hline HCP_A3 & $(\mathrm{Pd}, \mathrm{Y})_{1}(\mathrm{Va})_{5}$ & No excess term \\
\hline FCC_A1 & & ${ }^{0} \mathrm{~L}^{\mathrm{FCC} \_\mathrm{A} 1}=-640143+17.95 \mathrm{~T}$ \\
\hline $\mathrm{Pd}_{7} \mathrm{Y}$ & $(\mathrm{Pd})_{0.875}:(\mathrm{Y})_{0.125}$ & $\mathrm{G}_{\mathrm{Pd} \mathrm{Y}}^{\mathrm{Pd}}-0.875^{298} \mathrm{H}_{\mathrm{Pd}}^{\mathrm{FCC} \_\mathrm{A} 1}-0.125^{298} \mathrm{H}_{\mathrm{Y}}^{\mathrm{HCP} \_\mathrm{A} 3}$ \\
\hline \multirow{9}{*}{$\mathrm{Pd}_{3} \mathrm{Y}$} & & $=0.875 \mathrm{G}_{\mathrm{Pd}}^{\mathrm{FCC} \_\mathrm{A} 1}+0.125 \mathrm{G}_{\mathrm{Y}}^{\mathrm{HCP} \_\mathrm{A} 3}-54709+10.815 \mathrm{~T}$ \\
\hline & $(\mathrm{Pd})_{0.75}:(\mathrm{Y})_{0.25}$ & $\mathrm{G}_{\mathrm{Pd}: \mathrm{pd}}^{\mathrm{Pd} \mathrm{pd}_{0.25}}-\mathrm{H}_{\mathrm{Pd}}^{\mathrm{FCC} \_\mathrm{A} 1}=80000+\mathrm{GHSERPd}$ \\
\hline & & $\mathrm{G}_{\mathrm{Pd}: \mathrm{Y}}^{\mathrm{Pd}_{0.75} \mathrm{Y}_{0.25}}-0.75 \mathrm{H}_{\mathrm{Pd}}^{\mathrm{FCC}_{\mathrm{A} 1}}-0.25 \mathrm{H}_{\mathrm{Y}}^{\mathrm{HCP}-\mathrm{A} 3}$ \\
\hline & & $=+0.25$ GHSERPd +0.75 GHSERY -88887.7 \\
\hline & & $+6.135 \mathrm{~T}$ \\
\hline & & $\mathrm{G}_{\mathrm{Y}: \mathrm{Pd}}^{\mathrm{Pd}_{0.75} \mathrm{Y}_{0.25}}-0.75 \mathrm{H}_{\mathrm{Pd}}^{\mathrm{FCC}_{\mathrm{A} 1}}-0.25 \mathrm{H}_{\mathrm{Y}}^{\mathrm{HCP}-\mathrm{A} 3}$ \\
\hline & & $=+75000+0.75$ GHSERPd +0.25 GHSERY \\
\hline & & $+88887.7-6.135 \mathrm{~T}$ \\
\hline & & $\mathrm{G}_{\mathrm{Y}: \mathrm{Y}}^{\mathrm{Pd}_{0.75} \mathrm{Y}_{0.25}}-\mathrm{H}_{\mathrm{Y}}^{\mathrm{HCP} \_\mathrm{A} 3}=75000+\mathrm{GHSERY}$ \\
\hline \multirow[t]{2}{*}{$\mathrm{Pd}_{2} \mathrm{Y}$} & $(\mathrm{Pd})_{0.667}:(\mathrm{Y})_{0.333}$ & ${ }^{0} \mathrm{~L}_{\mathrm{Pd}: \mathrm{Y}, \mathrm{Pd}}^{\mathrm{Pd}_{0.72} \mathrm{Y}_{0.25}}=-103331.7-0.86 \mathrm{~T}$ \\
\hline & & $\mathrm{G}_{\mathrm{Pd} \cdot \mathrm{Y}}^{\mathrm{Pd}_{2} \mathrm{Y}}-0.667{ }^{298} \mathrm{H}_{\mathrm{Pd}}^{\mathrm{FCC} \_\mathrm{A} 1}-0.333{ }^{298} \mathrm{H}_{\mathrm{Y}}^{\mathrm{HCP} \_\mathrm{A} 3}$ \\
\hline \multirow[t]{2}{*}{$\mathrm{Pd}_{3} \mathrm{Y}_{2}-\mathrm{bt}$} & $(\mathrm{Pd})_{0.6}:(\mathrm{Y})_{0.4}$ & $=+0.667{ }^{298} \mathrm{H}_{\mathrm{Pd}}^{\mathrm{FCC} \_\mathrm{A} 1}+0.333{ }^{298} \mathrm{H}_{\mathrm{Y}}^{\mathrm{HCP} \_\mathrm{A} 3}-90302.78+3.7 \mathrm{~T}$ \\
\hline & & $\mathrm{G}_{\mathrm{Pd} \cdot \mathrm{Y}}^{\mathrm{Pd}_{3} \mathrm{Y}_{2}-\mathrm{bt}}-0.6 \mathrm{H}_{\mathrm{Pd}}^{\mathrm{FCC}_{\mathrm{A} 1}}-0.4 \mathrm{H}_{\mathrm{Y}}^{\mathrm{HCP} \_\mathrm{A} 3}$ \\
\hline $\mathrm{Pd}_{3} \mathrm{Y}_{2}$ ht & $(\mathrm{Pd})_{0.6}:(\mathrm{Y})_{0.4}$ & $=0.6 \mathrm{H}_{\mathrm{Pd}}^{\mathrm{FCC} \_\mathrm{A} 1}+0.4 \mathrm{H}_{\mathrm{Y}}^{\mathrm{HCP} \_\mathrm{A} 3}-90425+2.08 \mathrm{~T}$ \\
\hline \multirow[t]{2}{*}{$\mathrm{Pd}_{4} \mathrm{Y}_{3}$} & $(\mathrm{Pd})_{0.571}:(\mathrm{Y})_{0.429}$ & $\mathrm{G}_{\mathrm{Pd} \cdot \mathrm{Y}}^{\mathrm{Pd}_{3} \mathrm{Y}_{2} \text { ht }}-0.6 \mathrm{H}_{\mathrm{Pd}}^{\mathrm{FCC}_{\mathrm{A} 1}}-0.4 \mathrm{H}_{\mathrm{Y}}^{\mathrm{HCP} \_\mathrm{A} 3}$ \\
\hline & & $=0.6 \mathrm{G}_{\mathrm{Pd}}^{\mathrm{FCC} \_\mathrm{A} 1}+0.4 \mathrm{H}_{\mathrm{Y}}^{\mathrm{HCP} \_\mathrm{A} 3}-90107.45+1.84 \mathrm{~T}$ \\
\hline \multirow[t]{5}{*}{ PdY_bt } & $(\mathrm{Pd})_{0.5}:(\mathrm{Y})_{0.5}$ & $\mathrm{G}_{\mathrm{Pd} \cdot \mathrm{Y}}^{\mathrm{Pd}_{4} \mathrm{Y}_{3}}-0.571 \mathrm{H}_{\mathrm{Pd}}^{\mathrm{FCC}_{\mathrm{A} 1}}-0.429 \mathrm{H}_{\mathrm{Y}}^{\mathrm{HCP}_{\mathrm{A} 3}}$ \\
\hline & & $=0.571 \mathrm{G}_{\mathrm{Pd}}^{\mathrm{FCC} \_\mathrm{A} 1}+0.429 \mathrm{G}_{\mathrm{Y}}^{\mathrm{HCP} \_\mathrm{A} 3}-90509+1.493 \mathrm{~T}$ \\
\hline & & $\mathrm{G}_{\mathrm{Pd} \cdot \mathrm{Y}}^{\mathrm{Pd}_{0.5} \mathrm{Y}_{0.5}}-0.5 \mathrm{H}_{\mathrm{Pd}}^{\mathrm{FCC}_{\mathrm{A} 1}}-0.5 \mathrm{H}_{\mathrm{Y}}^{\mathrm{HCP}_{\mathrm{A} 3}}$ \\
\hline & & $=+0.5$ GHSERPd +0.5 GHSERY -91389.8748 \\
\hline & & $+3.16 \mathrm{~T}$ \\
\hline \multirow[t]{4}{*}{ PdY_ht } & $(\mathrm{Pd})_{0.5}:(\mathrm{Y})_{0.5}$ & $\mathrm{G}_{\mathrm{Y} \cdot \mathrm{Y}}^{\mathrm{Pd}_{0.5} \mathrm{Y}_{0.5}}-\mathrm{H}_{\mathrm{Y}}^{\mathrm{HCP} \mathrm{A}_{-} \mathrm{A} 3}=153095.5+\mathrm{GHSERY}$ \\
\hline & & ${ }^{0} \mathrm{~L}_{\mathrm{Pd} \cdot \mathrm{Y}: \mathrm{Y}}^{\mathrm{Pd}_{0.5} \mathrm{Y}_{0.5}}=-141542.47$ \\
\hline & & $\mathrm{G}_{\mathrm{Pd} \cdot \mathrm{Y}}^{\mathrm{Pd}_{0.5} \mathrm{Y}_{0.5}}-0.5 \mathrm{H}_{\mathrm{Pd}}^{\mathrm{FCC}_{\mathrm{Al}}}-0.5 \mathrm{H}_{\mathrm{Y}}^{\mathrm{HCP}-\mathrm{A} 3}$ \\
\hline & & $=+0.5$ GHSERPd +0.5 GHSERY -91389.8743 \\
\hline $\mathrm{Pd}_{2} \mathrm{Y}_{3}$ & $(\mathrm{Pd})_{0.4}:(\mathrm{Y})_{0.6}$ & $+3.16 \mathrm{~T}$ \\
\hline \multirow[t]{2}{*}{$\mathrm{Pd}_{2} \mathrm{Y}_{5}$} & $(\mathrm{Pd})_{0.286}:(\mathrm{Y})_{0.714}$ & $\mathrm{G}_{\mathrm{Y}: \mathrm{Y}}^{\mathrm{Pd}_{0.75} \mathrm{Y}_{0.25}}-\mathrm{H}_{\mathrm{Y}}^{\mathrm{HCP} \_\mathrm{A} 3}=+183383.5-7.7 \mathrm{~T}+\mathrm{GHSERY}$ \\
\hline & & ${ }^{0} \mathrm{~L}_{\mathrm{Pd}, \mathrm{Y}: \mathrm{Y}}^{\mathrm{Pd}_{0.5} \mathrm{Y}_{0.5}}=-162924.4$ \\
\hline \multirow[t]{6}{*}{$\mathrm{PdY}_{3}$} & $(\mathrm{Pd})_{0.25}:(\mathrm{Y})_{0.75}$ & $\mathrm{G}_{\mathrm{Pd} \cdot \mathrm{Y}}^{\mathrm{Pd}_{2} \mathrm{Y}_{3}}-0.4 \mathrm{H}_{\mathrm{Pd}}^{\mathrm{FCC}_{\mathrm{A} 1}}-0.6 \mathrm{H}_{\mathrm{Y}}^{\mathrm{HCP} \_\mathrm{A} 3}$ \\
\hline & & $=0.4 \mathrm{G}_{\mathrm{Pd}}^{\mathrm{FCC} \_\mathrm{A} 1}+0.6 \mathrm{G}_{\mathrm{Y}}^{\mathrm{HCP} \_\mathrm{A} 3}-81146.17+7.23 \mathrm{~T}$ \\
\hline & & $\mathrm{G}_{\mathrm{Pd}: \mathrm{Y}}^{\mathrm{Pd}_{2} \mathrm{Y}_{5}}-0.286 \mathrm{H}_{\mathrm{Pd}}^{\mathrm{FCC} \mathrm{C}_{\mathrm{A} 1}}-0.714 \mathrm{H}_{\mathrm{Y}}^{\mathrm{HCP} \_\mathrm{A} 3}$ \\
\hline & & $=0.286 \mathrm{G}_{\mathrm{Pd}}^{\mathrm{FCC} \_\mathrm{A} 1}+0.714 \mathrm{G}_{\mathrm{Y}}^{\mathrm{HCP} \_\mathrm{A} 3}-57513.8+3.47 \mathrm{~T}$ \\
\hline & & $\mathrm{G}_{\mathrm{Pd}: \mathrm{Y}}^{\mathrm{PdY}}-0.25 \mathrm{H}_{\mathrm{Pd}}^{\mathrm{FCC}_{\mathrm{A} 1}}-0.75 \mathrm{H}_{\mathrm{Y}}^{\mathrm{HCP}_{\mathrm{A} 3}}$ \\
\hline & & $=0.25 \mathrm{G}_{\mathrm{Pd}}^{\mathrm{FCC} \_\mathrm{A} 1}+0.75 \mathrm{G}_{\mathrm{Y}}^{\mathrm{HCP} \_\mathrm{A} 3}-50238.5+2.914 \mathrm{~T}$ \\
\hline
\end{tabular}


Table 2. Invariant reactions in the Pd-Y system.

\begin{tabular}{|c|c|c|c|c|}
\hline \multirow[t]{2}{*}{ Reaction } & \multicolumn{2}{|c|}{ Present work } & \multicolumn{2}{|c|}{$\operatorname{Ref}[7]$} \\
\hline & $\mathrm{T}(\mathrm{K})$ & $\mathrm{X}(\mathrm{Y})$ & $\mathrm{T}(\mathrm{K})$ & $\mathrm{X}(\mathrm{Y})$ \\
\hline Liq + BCC_A2 $\leftrightarrow$ HCP_A3 & 1751 & 0.980 & 1751 & 0.980 \\
\hline $\mathrm{Liq}+\mathrm{HCP} \_\mathrm{A} 3 \leftrightarrow \mathrm{PdY}_{3}$ & 1185 & 0.746 & 1181 & 0.745 \\
\hline $\mathrm{Liq} \leftrightarrow \mathrm{Pd}_{2} \mathrm{Y}_{5}+\mathrm{PdY}_{3}$ & 1183 & 0.734 & 1168 & 0.730 \\
\hline $\mathrm{Liq} \leftrightarrow \mathrm{Pd}_{2} \mathrm{Y}_{5}$ & 1187 & & 1188 & - \\
\hline $\mathrm{Liq} \leftrightarrow \mathrm{Pd}_{2} \mathrm{Y}_{5}+\mathrm{Pd}_{2} \mathrm{Y}_{5}$ & 1178 & 0.685 & 1176 & 0.700 \\
\hline Liq + PdY_ht $\leftrightarrow \mathrm{Pd}_{2} \mathrm{Y}_{5}$ & 1233 & 0.649 & 1233 & 0.660 \\
\hline Liq $\leftrightarrow$ PdY_ht & 1723 & & 1723 & 0.500 \\
\hline $\mathrm{Liq} \leftrightarrow \mathrm{PdY}_{2} \mathrm{ht}+\mathrm{Pd}_{4} \mathrm{Y}_{3}$ & 1658 & 0.453 & 1658 & 0.470 \\
\hline $\mathrm{Liq} \leftrightarrow \mathrm{Pd}_{4} \mathrm{Y}_{3}$ & 1683 & & 1683 & - \\
\hline $\mathrm{Liq}+\mathrm{Pd}_{4} \mathrm{Y}_{3} \leftrightarrow \mathrm{Pd}_{3} \mathrm{Y}_{2-}$ ht & 1546 & 0.376 & 1547 & 0.380 \\
\hline $\mathrm{Liq} \leftrightarrow \mathrm{Pd}_{3} \mathrm{Y}_{2-} \mathrm{ht}+\mathrm{Pd}_{2} \mathrm{Y}$ & 1519 & 0.367 & 1518 & 0.370 \\
\hline $\mathrm{Liq}+\mathrm{Pd}_{3} \mathrm{Y} \leftrightarrow \mathrm{Pd}_{2} \mathrm{Y}$ & 1578 & 0.342 & 1578 & 0.340 \\
\hline $\mathrm{Liq} \leftrightarrow \mathrm{Pd}_{3} \mathrm{Y}$ & 1973 & & 1973 & 0.250 \\
\hline \multirow[t]{5}{*}{$\mathrm{Liq} \leftrightarrow \mathrm{Pd}_{3} \mathrm{Y}+$ FCC_A1 } & 1478 & 0.147 & 1478 & 0.150 \\
\hline & & FCC & & \\
\hline & & 0.132 & & \\
\hline & & Pd3Y & & \\
\hline & & 0.225 & & \\
\hline \multirow[t]{4}{*}{ FCC_A1 $+\mathrm{Pd}_{2} \mathrm{Y} \leftrightarrow \mathrm{Pd}_{7} \mathrm{Y}$} & & FCC & & \\
\hline & 777 & 0.101 & 777 & 0.105 \\
\hline & & Pd3Y & & \\
\hline & & 0.224 & & \\
\hline $\mathrm{Pd}_{3} \mathrm{Y}_{2-} \mathrm{ht}$ & 1318 & 0.369 & 1318 & - \\
\hline PdY_ht $+\mathrm{Pd}_{2} \mathrm{Y}_{3} \leftrightarrow \mathrm{Pd}$ & 1171 & - & 1173 & 0.520 \\
\hline PdY_ht $\leftrightarrow \mathrm{Pd}_{4} \mathrm{Y}_{3}+\mathrm{PdY}_{-} \mathrm{b}$ & 1149 & - & 1143 & 0.510 \\
\hline
\end{tabular}

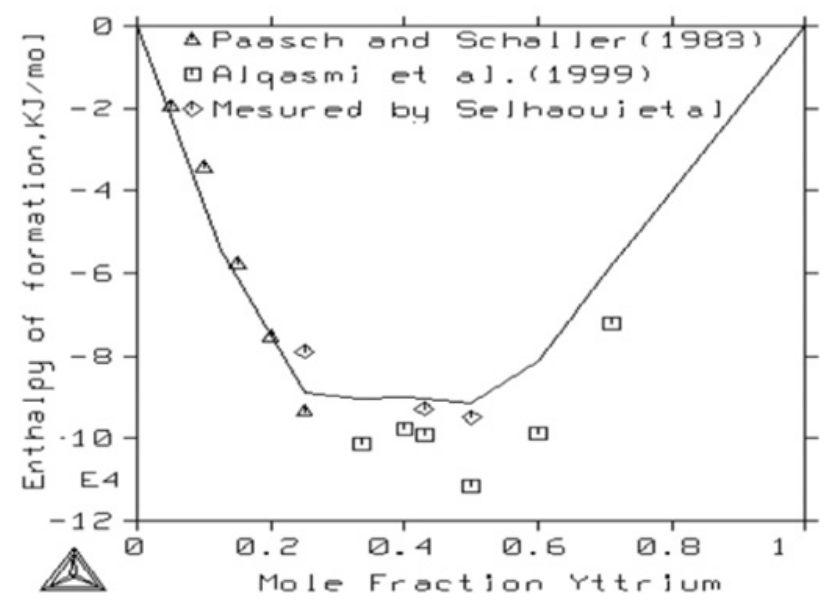

Figure 2. Calculated and measured enthalpies of formation of the intermetallic compounds.

errors (about 1-2 at. \%), all experimental invariant reaction compositions in the $\mathrm{Pd}-\mathrm{Y}$ system are well reproduced.

The assessed enthalpies of formation of the intermetallic compounds compared with experimental measurements are plotted in Fig. 2. The calculated enthalpies agree well with the experimental data [20-22].

As mentioned by [23] in order to check that the optimized thermodynamic parameters of the intermetallic compounds are satisfactory, we verified that when the liquid phase is suspended during the calculation of the Pd-Y phase diagram, the stoichiometric phases disappear

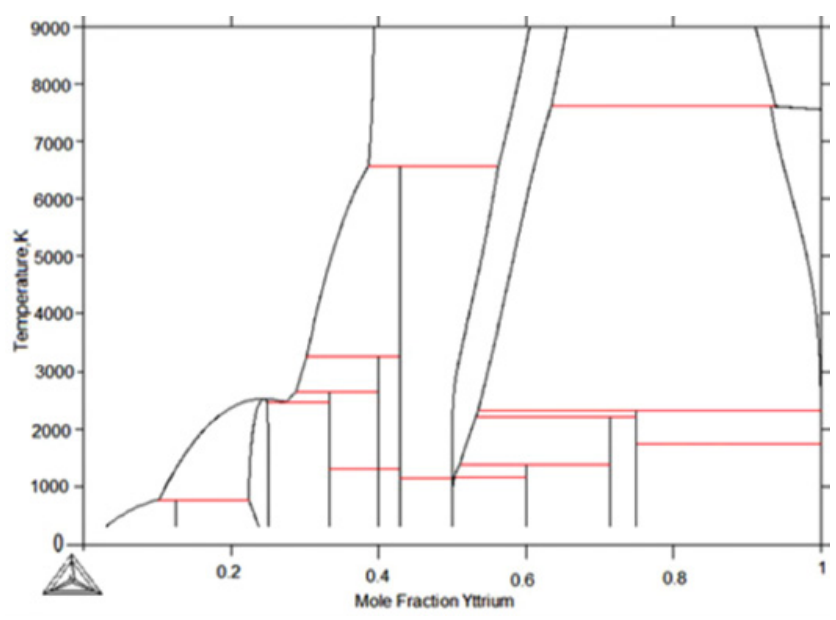

Figure 3. Calculated Pd-Y phase diagram when the liquid phase is suspended.

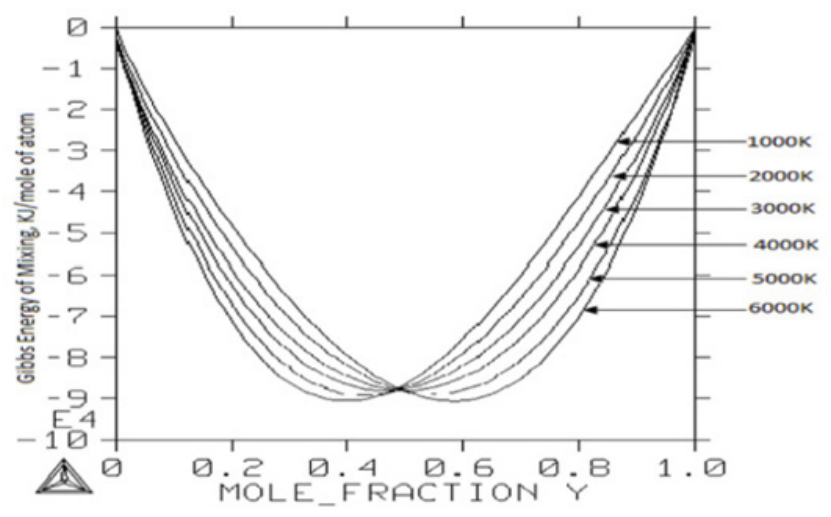

Figure 4. Gibbs energy of mixing of the liquid phase at different temperature.

at high temperatures. A terminal solid solutions and a two-phase domain existing between them are found to be stable (Fig. 3).

Figure 4 shows the evolutions of Gibbs energy for the liquid phase asa function of temperature $(\mathrm{T})$ with our optimization, when T is increasing up to $6000 \mathrm{~K}$, the Gibbs energyfor the liquid phase decreases.

The reference states were Pd and Y liquids.

\section{CONCLUSIONS}

All of the experimental phase equilibria and thermodynamic data of the Pd-Y system from the available literature have been critically evaluated. Within the regime of CALPHAD technique, the Redlich-Kister polynomial, the sublattice-compound energy model and the temperature dependant expression were adopted to describe the solution phases, the non-stoichiometric phases and the stoichiometric compounds, respectively. A set of self-consistent thermodynamic parameters has been obtained, which can reproduce satisfactorily most of the experimental data on thermodynamic properties and phase diagram within experimental uncertainties. 


\section{References}

[1] K.H.J. Buschow, Rep. Progr. Phys. 1977; 40: 11791256.

[2] L. Schlapbach, A. Züttel, Nature. 2001; 414: 353 358.

[3] V.K. Pecharsky, K.A. Gschneidner, Phys. Rev. Lett. 1997; 78: 4494-4497.

[4] L. Kaufman, H. Bernstein, Computer Calculation of Phase Diagram, Academic Press, New York, 1970.

[5] N. Saunders, A.P. Miodownik, CALPHAD - A Comprehensive Guide, Pergamon Press, Oxford, UK, 1998.

[6] H. Lukas, S.G. Fries, B. Sundman, Computational Thermodynamics - TheCalphad Method, Cambridge University Press, Cambridge, UK, 2007.

[7] Q. Loebich Jr., E. Raub, J. Less-Common Met. 1973; 30: 47-62.

[8] A. Palenzona, A. Iandelli, J. Less-Common Met. 1974; 34: 121-124.

[9] R. Sanjines-Zeballos, B. Chabot, E. Parthe, J. LessCommon Met. 1980; 72: 17-20.

[10] K. Takao, Y. Sakamoto, M. Yoshida, J. LessCommon Met. 1989; 152: 115-125.

[11] R. Kuentzler, O. Loebich, J. Less-Common Met. 1985; 106: 335-348.

[12] I.R. Harris, M. Norman, J. Less-Common Met. 1968; 15: 285-298.
[13] A. Palenzona, S. Cirafici, Thermochim. Acta.1975; 12: 267-275.

[14] G. Borzone, G. Cacciamani, R. Ferro, CALPHAD 1990; 14: 139-149.

[15] AT. Dinsdale, SGTE data for pure elements. Calphad 1991; 15: 317-425.

[16] O. Redlich, AKister. IndEng Chem. 1948; 40: 345-8.

[17] B. Sundman, B. Jansson, JO. Andersson. The Thermo-Calc databank system. CALPHAD. 1985; 9: 153-90.

[18] M. Hillert, L.I. Staffansson, Acta Chem. Scand. 1970; 24: 3618 .

[19] B. Sundman, J. Agren, J. Phys. Chem. Solids. 1981; 42: 297-301.

[20] S. Paasch, H.-J. Schaller, Ber. Bunsenges. Phys. Chem. 1983; 87: 812.

[21] R.A. Alqasmi, S. Paasch, H.J. Schaller, J. Alloys Comp. 1999; 283: 173-177.

[22] N. Selhaoui, O. J. Kleppa, J. Alloys Comp. 1993; 191.

[23] S.L. Chen, S. Daniel, F. Zhang, Y.A. Chang, W.A. Oates, R. Schmid-Fetzer, J. Phase Equilib. 2001; 22: 373.

[24] Binary Alloy Phase Diagrams, Second Edition, Ed. T.B. Massalski, ASM International, Materials Park, Ohio. 1990; 3: 3065-3068. 